\title{
Autour de l'estropié du Musée d'art et d'histoire de Genève
}

\author{
Une représentation archaïque grecque d'hémimélie?*
}

Véronique Dasen

\section{Summary}

An archaic Greek terracotta vase in the Art and History Museum at Geneva depicts a man deprived of his left arm and with two legs ending in a stump below the knees. Did he suffer from a traumatism (amputation), a mutilating disease or a congenital malformation (hemimelia)? A survey of written and iconographic sources throws light on the methods and limits of ancient surgery, and on the chances of survival of abnormal children in archaic and classical Greece.

\section{Résumé}

Le Musée d'art et d'histoire de Genève possède un vase grec en terre cuite de l'époque archaïque figurant un homme privé du bras gauche et dont les membres inférieurs se terminent par des moignons au niveau des genoux. S'agit-il d'un personnage ayant subi un traumatisme (amputation), atteint d'une maladie mutilante ou d'une malformation congénitale (hémimélie)? Un examen des sources écrites et iconographiques permet de faire le point sur les méthodes et les limites de la chirurgie antique, ainsi que sur les chances de survie des nouveau-nés atteints d'anomalies physiques en Grèce archaïque et classique.

\section{* Abréviations:}

CVA Corpus vasorum antiquorum

LIMC Lexicon Iconographicum Mythologiae Classicae, Zürich et München, 1981

Sauf mention contraire, toutes les traductions citées ici sont tirées des éditions Belles-Lettres à Paris

Dr. phil. Véronique Dasen, Le Hobelet, 1783 Pensier 


\section{L'estropié du Musée d'art et d'histoire de Genève}

La tératologie, c'est-à-dire l'étude des anomalies et des monstruosités, est un domaine que la médecine antique n'a que rarement abordé. Les Anciens ontils reconnu l'existence de malformations congénitales? Ont-ils eu la possibilité d'observer des nouveau-nés ou même des adultes atteints de graves anomalies physiques? Comment ont-ils tenté d'expliquer la formation de telles malformations? Les sources antiques ne permettent pas de répondre aisément à ces questions. Les documents littéraires et iconographiques relatifs aux anomalies physiques chez l'homme sont rares et disparates.

Cet article propose d'apporter quelques éléments de réponse en présentant le témoignage d'un objet exceptionnel conservé au Musée d'art et d'histoire de Genève (fig. $1 \mathrm{a}-\mathrm{d})^{1}$. Il s'agit d'un vase plastique en terre cuite de l'époque archaïque. Il figure un homme nu, assis, dont le bras gauche manque, tandis que ses deux membres inférieurs se terminent par des moignons au niveau des genoux; son bras droit est allongé le long du corps, la paume de la main appuyée sur le sol. Un petit orifice sur le sommet de la tête constitue l'ouverture du vase. La partie droite du visage est brisée, et deux éclats manquent dans le cou. La figurine aurait été découverte en Sicile, plus précisément dans la nécropole de Sélinonte. Elle aurait été trouvée dans un dinos en bronze martelé qui servait d'urne cinéraire ${ }^{2}$. Une deuxième pièce identique, provenant du même moule, mais fragmentaire, est conservée dans une collection privée en Suisse ${ }^{3}$.

Ce vase plastique provient d'un atelier corinthien, comme l'indique la qualité très fine de l'argile de couleur brun clair. Divers éléments stylistiques renforcent cette attribution, comme la façon de rendre la pilosité par de petits points bruns, ici sur la face (sous la lèvre inférieure), sur le torse, le pubis et les bourses.

Les parallèles les plus proches se trouvent dans la série de vases plastiques corinthiens figurant des hommes accroupis, conventionnellement appelés «comastes». Dès le VI ${ }^{\mathrm{e}} \mathrm{s}$. av. J.-C. ces vases sont généralement moulés, avec un orifice discrètement placé sur le sommet du crâne, comme sur le vase de

$1 \mathrm{~N}^{\circ}$ inv. HR 79. H. 9,5 cm, L. 5,7 cm. J. Dörig, Art antique, Genève, 1975, $\mathrm{n}^{\circ} 143$; Médecine antique. IV $V^{e}$ colloque international hippocratique, Lausanne, 1981,91-92, $\mathrm{n}^{\circ} 61$; G. Python, Les vases plastiques du Musée d'art et d'histoire de Genève, Mémoire de licence, Université de Genève, mai 1994, 40-42 (inédit).

2 Le dinos est en cours de publication (P. Birchler, Université de Genève).

3 Seule la tête et une partie du haut du corps sont conservés. Je remercie J.-L. Zimmermann pour cette information.

4 Cf. J. Ducat, «Les vases plastiques corinthiens», Bulletin de Correspondance Hellénique 87, 1963, 431-458. En particulier, voir le phallus corinthien entièrement moucheté conservé à Bonn 510; M. I. Maximova, Les vases plastiques dans l'antiquité, Paris, 1927, n 144, pl. 39. 

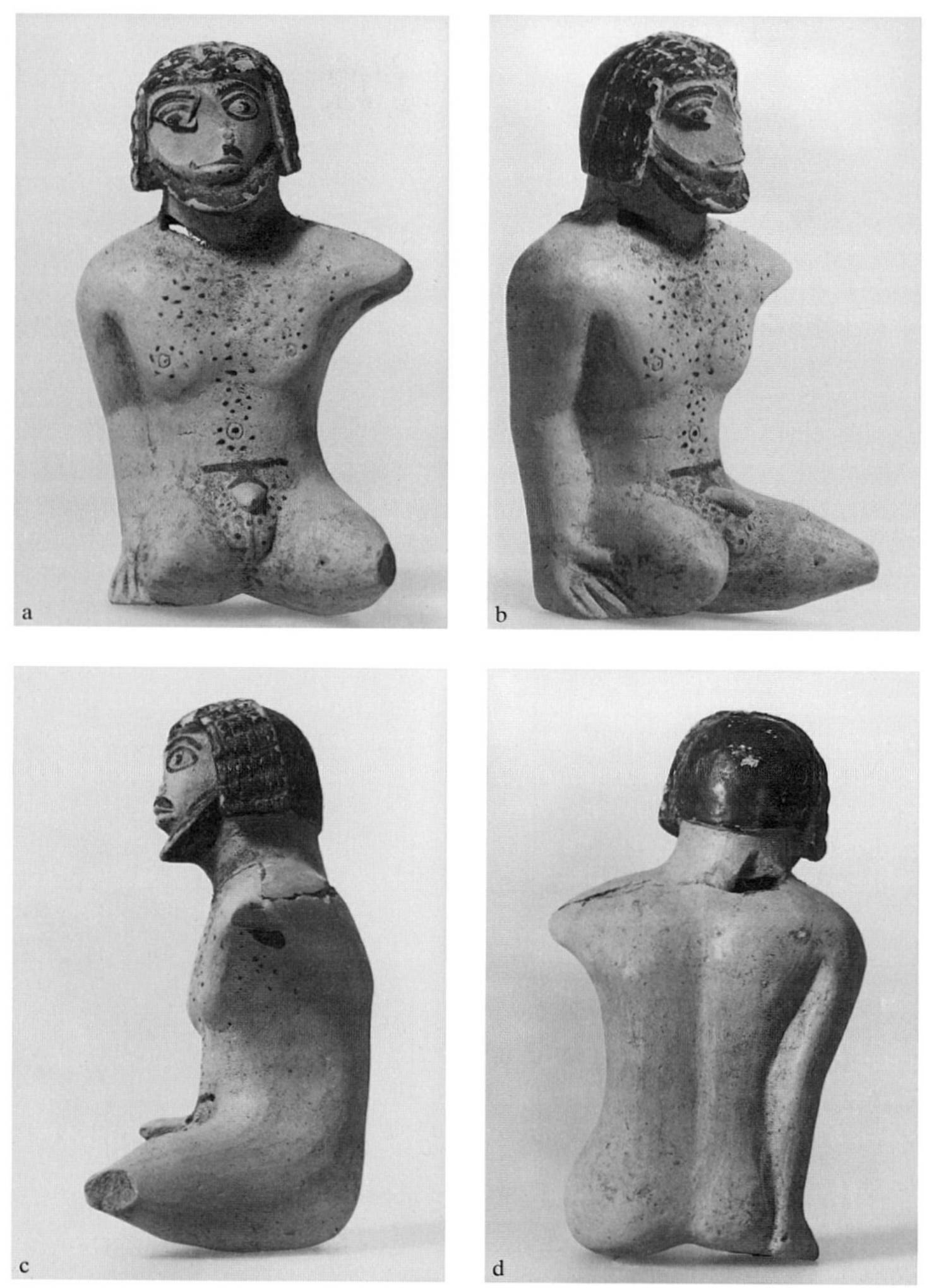

Fig. 1 a-d. Terre cuite. Genève, Musée d'art et d'histoire HR 79. Photo V. Siffert. 

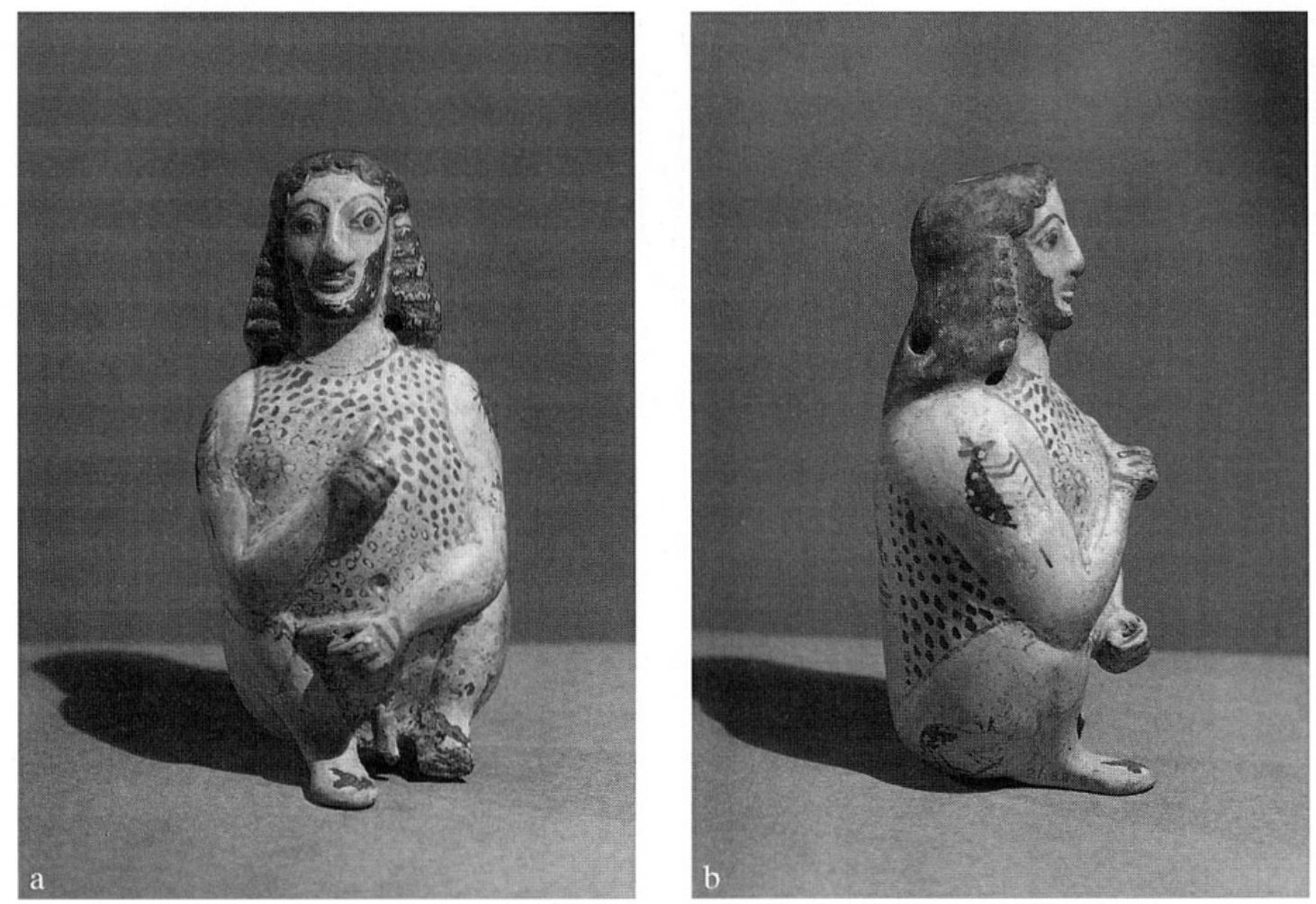

Fig. 2 a, b. Terre cuite. New York, Metropolitan Museum of Art 21.88.8 (Rogers Fund 1921). Photo New York MMA.

Genève. Les comastes présentent les mêmes traits expressifs du visage, avec de grands yeux en amande, exorbités, et d'épais sourcils parallèles aux paupières supérieures; ils portent souvent une moustache et une barbe plate à arête aiguë peintes en noir ${ }^{5}$. En particulier, les comastes du groupe de Mégara Hyblaea (fig. 2 a, b) offrent des similitudes supplémentaires avec l'estropié de Genève: dans les deux cas, les cheveux des personnages sont incisés sur la partie antérieure de la tête pour figurer des nattes ou des boucles, tandis qu'à l'arrière ils forment une masse lisse. Notre vase date probablement de la même période que les comastes de Mégara Hyblaea, c'est-à-dire du premier quart du $\mathrm{VI}^{\mathrm{e}}$ s. av. J.-C. ${ }^{6}$

L'examen de la série des comastes met en évidence l'originalité de la figurine de Genève. L'artiste a su se démarquer des conventions stylistiques contemporaines pour créer une œuvre d'un réalisme saisissant.

La pose du personnage n'a ainsi pas de parallèle comparable dans la série des comastes: ceux-ci sont généralement accroupis, les jambes étroitement

5 Sur cette série, voir Ducat (n. 4 supra); K. Wallenstein, Korinthische Plastik des 7. und 6. Jahrhunderts vor Christus, Bonn, 1971; R. A. Higgins, Catalogue of the terracottas in the Department of Greek and Roman Antiquities, British Museum, II, London, 1975, 37-39.

6 Ducat (n. 4 supra) 447, $\mathrm{n}^{\text {os }} 2-4$, figs. 15-17 (540 av.); Wallenstein (n. 5 supra) 133-134, ${ }^{\text {os }} 12-14$, pl. 15. Ducat propose la datation de 540 av. J.-C., Wallenstein de 580/570 av. J.-C. 
collées au corps, les coudes appuyés sur les genoux, les poings serrés ramenés sur la poitrine, tandis que l'estropié est assis, les jambes clairement détachées l'une de l'autre, le bras droit accolé au corps'. Le corps des comastes, grotesquement pansu, donne au vase une forme ovoïde; les détails anatomiques, comme le nombril, ne sont que grossièrement indiqués ${ }^{8}$; le torse de l'estropié est par contre soigneusement modelé, avec l'indication d'un développement musculaire plus important du côté droit; les mamelons et le nombril sont marqués par des cercles incisés entourés de petits points. La coiffure de l'estropié diffère aussi de celle des comastes: sa coupe rectangulaire est de style dédalique, les cheveux sont très courts et forment une masse compacte qui cache les oreilles ${ }^{9}$. Le décor peint des vases plastiques contraste également avec la nudité de l'estropié:le corps des comastes peut être entièrement recouvert de petits points évoquant un pelage semblable à celui des satyres ${ }^{10}$, orné de motifs géométriques, floraux ou animaux ${ }^{11}$, et même parfois revêtu d'une tunique mouchetée et de bottines évoquant le costume des «danseurs rembourrés» de la céramique corinthienne (fig. $2 \mathrm{a}, \mathrm{b})^{12}$. Ces décors font allusion soit à l'association des comastes au monde dionysiaque (toison de satyre, nébride), soit à leur caractère apotropaïque. La figurine de Genève est, elle, dépourvue d'allusions au monde surnaturel; son réalisme anticipe celui des terres cuites anatomiques de l'époque hellénistique. La seule fantaisie que le coroplaste semble s'être permise est de mettre en évidence le sexe du personnage d'un trait de pinceau sur le pubis, comme pour évoquer les vases plastiques contemporains en forme de phallus ${ }^{13}$.

L'infirmité de l'estropié soulève de nombreuses questions, d'une part d'ordre médical, liées à l'établissement d'un diagnostic rétrospectif: son handicap est-il congénital ou acquis? Les sources littéraires et iconographiques ont-elles conservé la trace de cas comparables? Comment a-t-on tenté d'expliquer rationnellement l'origine de telles anomalies? Des interrogations, d'autre part, relatives à l'histoire des mentalités: Un nouveau-né atteint d'une malformation aussi spectaculaire aurait-t-il pu survivre? Pourquoi un

7 Exception: le bras droit est allongé le long du corps chez un comaste conservé à Munich, Antikensammlungen, 6633 (deuxième quart du VII ${ }^{\mathrm{e}}$ s. av. J.-C.); $C V A$ München 3, pl. 148,1-4; Wallenstein (n. 5 supra) $95, \mathrm{n}^{\circ} 2$, pl. 1, 3-5.

8 Voir p. ex. le comaste conservé à Paris, Louvre, CA 1631; CVA Louvre 8 pl. 1, 4-6.

$9 \mathrm{Cf}$. la coupe de cheveux similaire de la figurine de Munich (n. 7 supra) et de Londres, British Museum, A 1100; Higgins (n. 5 supra) p. 38, n 1664, pl. 26; Wallenstein (n. 5 supra), 95, nº 1 , pl. 1, 1-2.

10 Voir p. ex. CVA Musée Scheurleer 1, pl. 1,5.

11 Voir p. ex. Ducat (n. 4 supra) 442 no 16 fig. 11 (motifs géométriques, oiseau); CVA Louvre 8 pl.1, 4-6 (œil prophylactique).

12 Higgins (n. 5 supra) $38-39$, nos 1665,1666 et 1667, pls $26-27$; CVA Louvre 8 pls 3 et 4, 1-8.

13 Comme le note Dörig (n. 1 supra), le pubis «ressemble à la partie supérieure d'un alabastron». Cf. Higgins (n. 5 supra) 42-43, n 1676 pl. 29. 
coroplaste aurait-il représenté un estropié? Quelle est la signification de cette figurine dans un contexte funéraire?

\section{Aspects médicaux}

Trois types de possibilités doivent être envisagées: l'estropié peut représenter les effets d'un traumatisme, accidentel ou volontaire (a), d'une maladie (b), ou d'une malformation congénitale (c).

\section{a) Traumatisme}

Une mutilation peut avoir différentes origines. Involontaire, si elle résulte d'un accident, d'une blessure de guerre ou de chasse. Volontaire, si elle est due à une opération chirurgicale à motif thérapeutique (pour lutter contre une gangrène par exemple); il peut également s'agir d'une amputation punitive, d'un châtiment infligé à un criminel ou un prisonnier.

L'hypothèse de l'amputation nous amène à nous poser la question de l'état des connaissances médicales à l'époque archaïque. Une opération de cette envergure était-elle alors concevable?

Pour réaliser une amputation dans de bonnes conditions, trois conditions doivent être réunies: la maîtrise de l'hémorragie (l'hémostase), le contrôle de la douleur (l'anesthésie) et des infections (l'asepsie). Le problème de l'hémostase est le plus important. Un examen des fragments de traités médicaux pré-hippocratiques nous révèle des conceptions anatomiques encore sommaires. Le fonctionnement du système cardio-vasculaire est mal connu; pour Alcméon de Crotone (vers 500 av. J.-C.), les vaisseaux sanguins proviennent de la tête et les artères ne contiennent que de l'air ${ }^{14}$. Les moyens utilisés pour stopper un saignement accidentel étaient probablement les mêmes que ceux décrits par les médecins hippocratiques: il s'agit essentiellement d'applications de compresses froides et de styptica, d'élévation du membre, et plus rarement, de cautérisation ${ }^{15}$. Faute de savoir maîtriser les hémorragies, la chirurgie pré-hippocratique se limite à l'extraction de projectiles et à la réduction de fractures et luxations.

14 Voir p. ex. C. R. S. Harris, The heart and the vascular system in ancient Greek medicine from Alcmaeon to Galen, Oxford, 1973,3-9 (Alcméon).

15 S. C. Harvey, «The history of hemostasis», Ann. Med. Hist. New ser. 1, 1929, 127-154; R. von Grot, «Über die in der hippokratischen Schriftsammlung enthaltenen pharmakologischen Kenntnisse», in Historische Studien zur Pharmakologie der Griechen, Römer und Araber, Leipzig, 1968,117-122. Voir p. ex. Epidémies 6,7,2 = Littré 5, 337-339 (compresses, bandages); Des lieux dans l'homme 40 = Littré 6, 331 (cautérisation); Aphorismes 5,23 = Littré 4, 541 (compresses froides). On retrouve les mêmes procédés chez Celse 5,26,21. 
La médecine hippocratique elle-même a une attitude essentiellement conservatrice; le médecin se contente de suivre le processus gangréneux et d'aider le membre à se détacher quand les tissus sont suffisamment nécrosés et insensibles ${ }^{16}$. L'auteur du traité Des articulations met clairement en garde contre une tentative d'amputation prématurée qui pourrait entraîner la mort du patient suite à une hémorragie ou au choc de la douleur ${ }^{17}$.

La chirurgie fait des progrès majeurs à l'époque hellénistique, entre autres grâce à la technique de la ligature des vaisseaux sanguins, probablement découverte par les médecins d'Alexandrie ${ }^{18}$. Dans le De Medicina $\left({ }^{\mathrm{er}} \mathrm{s}\right.$. apr. J.-C.), Celse décrit l'ablation de membres au-dessus de la zone gangreneuse; l'hémorragie est contrôlée en recourant soit à la ligature artérielle, soit à des moyens traditionnels, comme la compression ou la cautérisation ${ }^{19}$. Mais de telles opérations semblent n'avoir été pratiquées qu'en cas d'extrême urgence; Celse relève que l'exercice est périlleux, «car souvent, au milieu même de l'opération, le malade meurt d'hémorragie ou de syncope ${ }^{20}$, sans oublier les risques de complications infectieuses ${ }^{21}$. Chez Paul d'Egine (VII ${ }^{e}$ s. apr. J.-C.), l'emploi de la ligature artérielle n'est toujours pas systématique et l'amputation reste une opération redoutée ${ }^{22}$. L'incompréhension du système sanguin pouvait de surcroît entraîner des décisions dangereuses, comme celle de laisser saigner la blessure pour éviter une infection malgré le danger mortel que l'hémorragie faisait encourir au patient.

Le manque de contrôle efficace de la douleur a également limité le développement de la chirurgie antique. Les Anciens connaissaient divers produits d'origine végétale ou minérale pour soulager la douleur, comme la poudre de marbre de Memphis, que l'on utilisait en application, mélangée à du vinaigre, pour endormir localement les parties qu'on voulait couper ou cautériser ${ }^{23}$. On reconnaissait à des plantes, comme la mandragore, un effet analogue; Pline raconte qu'«on prend leur suc contre les morsures de ser-

16 G. Majno, The healing hand. Man and wound in the ancient world, Cambridge Mass., 1975, 191-192, 328.

17 Hippocrate, Des articulations $69=$ Littré 4, 283-289.

18 Majno (n. 16 supra) 328-329; M. Michler, Die alexandrinischen Chirurgen, Wiesbaden, 1968, 6-7, 11-14, 37.

19 Voir p. ex.5,26,21 (avec ligature des vaisseaux) 7,33 (cautérisation). Cf. Galien, De la méthode thérapeutique 2,11 (cautérisation). Sur ces progrès, voir Majno (n. 16 supra) 362-365,403-404. Sur l'hémostase chez Galien: N. Mani, «Die wissenschaftlichen Grundlagen der Chirurgie bei Galen», in F. Kudlien, R. J. Durling (eds.), Galen's method of healing. Proceedings of the 1982 Galen symposium, Leiden etc., 1991,34-47.

20 Trad. M. Nisard, Paris, 1846.

21 Voir p. ex. Celse 5,26,22; M. D. Grmek, Les maladies à l'aube de la civilisation occidentale, Paris, 1983, 190-192 (gangrènes mortelles).

22 Cf. Paul d'Egine, Chirurgie (ed. R. Briau, Paris, 1855), ch. 43 (amputation de doigts surnuméraires), ch. 84 (amputation des extrémités, cautérisation).

23 Plin. Nat. 36,56; Dioscorides 5,140. 
pents, ainsi qu'avant de souffrir l'amputation de quelque partie du corps, afin de s'engourdir contre la douleur» ${ }^{24}$. Les propriétés soporifiques du pavot étaient aussi bien connues; Pline décrit comment récolter le suc du pavot noir en incisant la plante sous la tête ou le calice. Mais aucun texte ne mentionne son usage régulier à des fins médicales. Pline relève que l'opium n'est pas un soporifique inoffensif, il peut aussi faire mourir pendant le sommeil s'il est pris à trop forte dose: «aussi l'opium est-il l'objet de grands débats. Diagoras et Erasistrate l'ont absolument condamné comme un poison mortel» ${ }^{25}$.

Un examen des sources littéraires confirme l'extrême rareté de telles opérations. Quelques textes mentionnent l'amputation d'un membre et l'usage de prothèses. Hérodote $(9,37)$ rapporte ainsi qu'en 479 av. J.-C. Hégésistrate d'Elis, capturé par les Spartiates, se trancha le pied pour se libérer de l'entrave qui le maintenait prisonnier; réfugié à Tégée, il guérit de sa blessure et se fit fabriquer un pied artificiel en bois; c'est muni de cette prothèse qu'il servit de devin au Perse Mardonios et fut associé à la bataille de Platée. Au $\mathrm{II}^{\mathrm{e}}$ s. apr. J.-C., Lucien raconte l'histoire, réelle ou inventée, d'un riche d'Asie qui eut les deux pieds amputés à la suite de gelures. Il se fit faire des pieds en bois, mais avec lesquels il ne pouvait pas marcher sans l'aide de ses esclaves, ce qui ne l'empêcha pas «d'acheter continuellement des chaussures neuves et magnifiques» ${ }^{26}$. Une autre anecdote concerne l'ablation d'une main: à la fin du III ${ }^{\mathrm{e}}$ s. av. J.-C., Marcus Sergius Silus perdit sa main droite au combat. Selon Pline, il se fit fabriquer une main en fer et participa encore à plusieurs batailles $^{27}$. On relèvera que dans tous ces exemples l'amputation ne concerne que l'extrémité des membres et non les membres entiers; on parle encore moins de plusieurs membres amputés.

Les cultures disposant de moyens analogues à ceux de la médecine grecque archaïque ne connaissent pas non plus d'opérations de ce genre. On y pratique essentiellement des trépanations ou des mutilations rituelles ou punitives touchant les extrémités, en particulier les mains, ou les parties molles comme le nez ou les oreilles ${ }^{28}$. Là-aussi, les problèmes posés par la maîtrise de l'hémorragie, l'absence d'asepsie et de moyens anesthésiques semblent avoir constitué des obstacles majeurs. Les chirurgiens Massaï représentent une exception: malgré l'absence d'anesthésie et une asepsie

24 Plin. Nat. 25,149-150; Dioscorides 4,75.

25 Plin. Nat. 20,198-200. Voir M.L. Baur, «Recherches sur l'histoire de l'anesthésie avant 1846», Janus 31, 1927, 24-90, 124-182, 213-270. En particulier: 180-182, 213 (opium);264-270 (mandragore); 135, 223-225 (poudre du Caire).

26 Lucien, Contre un ignorant bibliomane, 6 (trad. E. Talbot, 1857).

27 Plin. Nat. 7,104-106.

28 E. H. Ackerknecht, «Primitive surgery», in D. R. Brothwell, A.T. Sandison (eds.), Diseases in antiquity, Springfield, 1967, 635-650. Pour l'Egypte pharaonique: A.-P. Leca, La médecine au temps des pharaons, Paris, 1988, 433-434. 
rudimentaire, ils savent amputer des membres et fabriquent même des prothèses; leur réussite tient au fait qu'ils pratiquent la ligature artérielle ${ }^{29}$.

Les spécimens ostéo-archéologiques sont rares et difficiles à identifier avec certitude. D. R. Brothwell et V. Moller-Christensen décrivent un cas d'amputation de l'avant-bras droit, avec guérison du moignon, sur des ossements provenant d'Egypte et datant de 2000 av. J.-C ${ }^{30}$. Un squelette découvert à Capoue dans une tombe datant de $300 \mathrm{av}$. J.-C. avait la partie inférieure de la jambe droite remplacée par une prothèse artificielle en bois recouverte de feuilles de bronze ${ }^{31}$.

L'estropié du Musée d'art et d'histoire n'a donc probablement pas été amputé. Cette hypothèse peut être exclue en l'absence de connaissances anatomiques, techniques et pharmaceutiques suffisantes à l'époque archaïque. Le patient n'aurait pas survécu à l'ablation d'un ou plusieurs membres; en l'absence de recours à la ligature artérielle, il aurait succombé à une hémorragie $^{32}$.

\section{b) Maladie}

Quelques maladies infectieuses peuvent entraîner des lésions locales du squelette et causer la perte d'un membre ou d'une partie de membre, comme la tuberculose osseuse, l'ostéomyélite ou la lèpre; un diabète peut également provoquer une gangrène ${ }^{33}$. Mais ces maladies progressent de façon très localisée et ne touchent que les extrémités et les parties molles. L'hypothèse de la lèpre peut être clairement éliminée: le visage de l'estropié de Genève ne porte aucune trace des lésions mutilantes caractéristiques de la maladie; de plus, la lèpre est très rare dans le monde grec avant l'époque hellénistique $^{34}$.

29 M. Merker, Die Masai, Berlin, 1910, 187-196; Ackerknecht (n. 28 supra) 636, 640, 647.

30 D. R. Brothwell,V.Moller-Christensen, «A possible case of amputation, dated to c. 2000 B.C.», Man 63, 1963, 192-194; D. J. Ortner, W. G. J. Putschar, Identification of pathological conditions in human skeletal remains, Washington, 1981, 94-95 (mettent en doute le diagnostic de 1963).

31 London, Science Museum, inv. ${ }^{\circ}$ A646752 (facsimile; l'original a été détruit pendant la deuxième guerre mondiale). Voir L. J. Bliquez, «Prosthetics in Classical Antiquity: Greek, Etruscan, and Roman prosthetics», Aufstieg und Niedergang der römischen Welt, 37/3, 1996, 2640-2676, en particulier 2667-2673, pls XIX-XXII.

32 Sur l'importance de la ligature artérielle lors du processus d'amputation, voir D. J. Larrey, Mémoires de chirurgie militaire et histoire de ses campagnes, 4 vols, Paris, 1812-1817, et en particulier son «Mémoire sur les amputations» (vol. 2, 451-506). La technique de l'amputation ne progresse qu'à partir du XVI ${ }^{\mathrm{e}} \mathrm{s}$. (Ambroise Paré) avec le développement de la chirurgie militaire suite à l'invention des armes à feu. Cf. Briau (n. 22 supra) 13-14.

33 Ortner/Putschar (n. 30 supra) 105-129 (ostéomyélite), 141-176 (tuberculose osseuse), 176-180 (lèpre); Grmek (n. 21 supra), 227-260 (lèpre).

34 Grmek (n. 21 supra) 249-252, 258-260. 


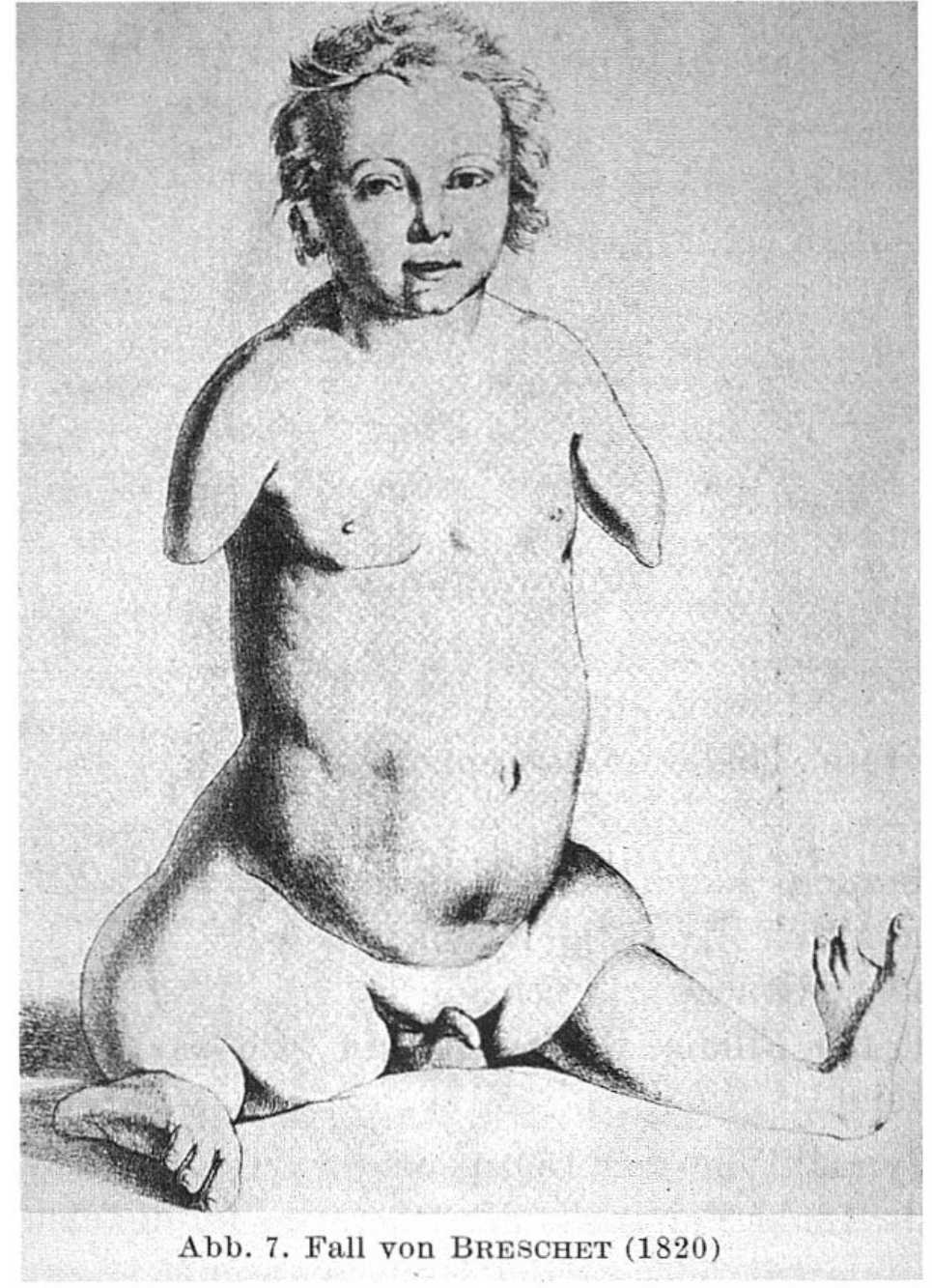

Fig. 3. Cas de Breschet, 1820. D'après D. Kühne et al., Humangenetik 3, 1967, 250, $\mathrm{n}^{\circ} 14$, fig. 7 .

\section{c) Malformation congénitale}

La terre cuite de Genève pourrait figurer une malformation congénitale, et, plus précisément, un cas de dysmélie, une anomalie touchant les membres ${ }^{35}$. Parmi les nombreuses formes connues de dysmélie, l'ectromélie pourrait s'appliquer à notre personnage. Cette anomalie, très rare, est caractérisée par un défaut de développement, total ou partiel, d'un ou plusieurs membres ou segments de membre. Quand les quatre membres sont atteints, l'ectromélie est totale. On parle alors d'amélie, ou, dans le langage populaire, d'«homme-

35 W. J.W. Sharrard, «Major congenital reduction deformities of the limbs», in Paediatric orthopaedics and fractures, Oxford etc., 1979, ${ }^{2}$ 208-276; L. Henkel, H.-G. Willert, «Dysmelia: a classification and a pattern of malformation in a group of congenital defects of the limbs», J. Bone Joint Surg. 51B, 1969, 399-414; W. Lenz, U. Feldmann, «Unilateral and asymetric limb defects in man: delineation of the femur-fibula-ulna complex», in Birth defects: original article series, Morphogenesis and malformation of the limb, 13, $\mathrm{n}^{\circ}$ 1, New York, 1977,269-285. M. P. Balard, «Une famille de phocomèles», Journal de médecine de Bordeaux et du Sud-Ouest 114/ $\mathrm{n}^{\text {os }} 30-31,1937,115-116$, rapporte l'observation de la transmission de malformations des membres supérieurs sur quatre générations d'une même famille (phocomélie et ectromélie). 


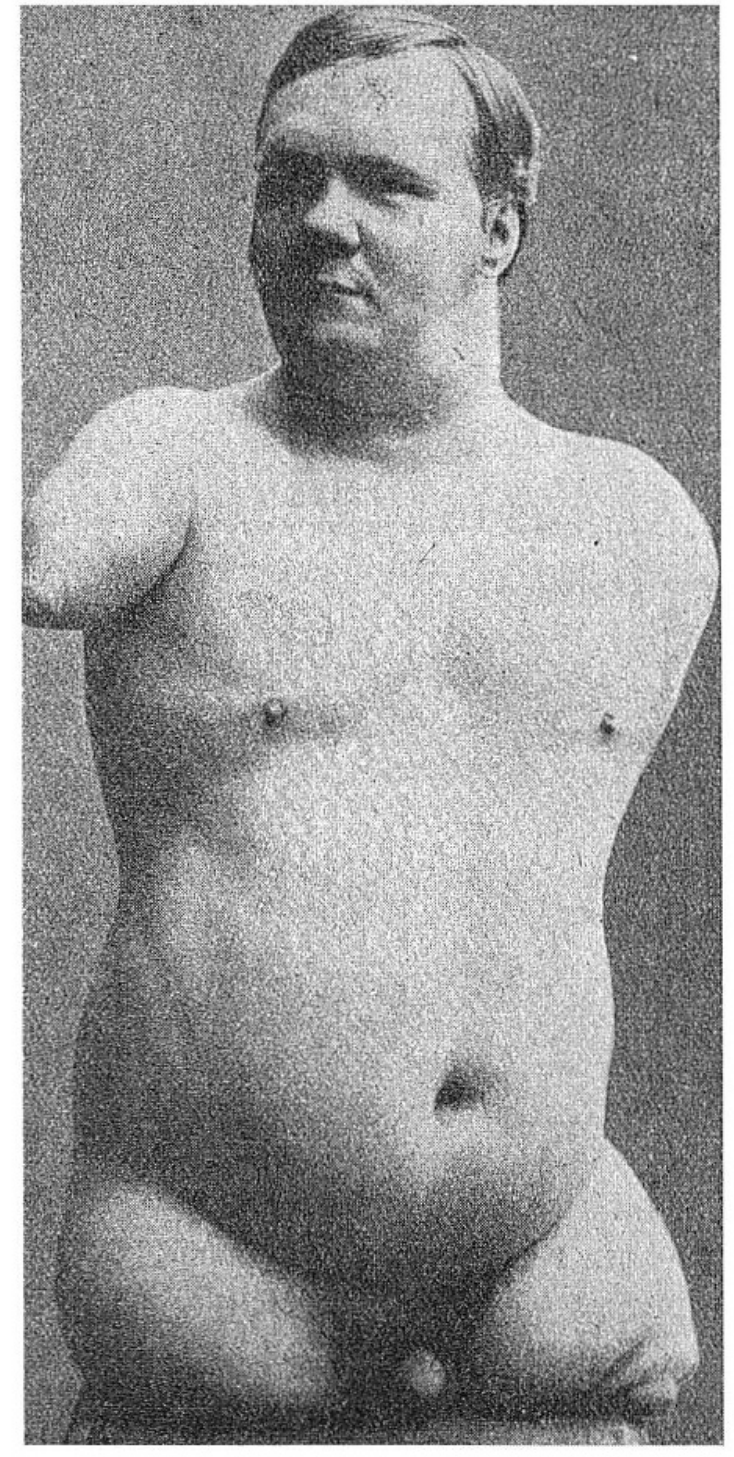

Fig. 4. N. Vassilievitch Kobelkoff, né en 1851 en Sibérie. D'après G.-J. Witkowski, Curiosa de médecine, Paris, 1920,139-140, fig. 47.

tronc». Quand les segments proximaux sont seuls atteints, la main ou le pied s'attache directement sur le tronc: il s'agit de phocomélie, une anomalie mieux connue par les méfaits causés par la thalidomide dans les années 1960. L'estropié de Genève pourrait représenter un cas d'hémimélie, une forme distale d'ectromélie, qui atteint les membres à partir du genou ou du coude (figs 3-4) ${ }^{36}$. Elle toucherait ici les deux jambes et serait accompagnée d'une absence totale du membre supérieur gauche. Relevons que l'hémimélie est souvent associée à l'anomalie d'autres membres, comme l'agénésie ou absence de doigts de la main ou du pied, dit «en fourche» ou «en pince de homard» ${ }^{37}$.

36 I. Geoffroy Saint-Hilaire, Histoire générale et particulière des anomalies de l'organisation chez l'homme et les animaux, II, Paris, 1836, 231-232; Lenz/Feldmann (n. 35 supra) 277 fig. 13.

37 Cf. Lenz/Feldmann (n. 35 supra) 272-273, figs 6-7. La syndactylie (fusion de deux doigts) est également parfois associée à une malformation des membres; D. Kühne et al., «Defekt von Femur und Fibula mit Amelie, Peromelie oder ulnaren Strahldefekten der Arme», Human- 
L'estropié est peut-être atteint d'une anomalie secondaire de ce type; sa main a une apparence anormale: on ne distingue que quatre doigts, dont le pouce qui a une forme et une position inhabituelles. Un autre détail parle en faveur d'une malformation congénitale: on remarque que le muscle pectoral droit est très développé, une hypertrophie qui pourrait résulter de l'usage exclusif du bras droit. La colonne vertébrale ne montre pas de déformation compensatoire, mais la position de la tête, légèrement inclinée vers la droite, pourrait l'évoquer.

\section{Des cas similaires dans les sources littéraires et iconographiques}

Des cas similaires sont-ils décrits dans les sources littéraires? Seuls quelques textes scientifiques antiques parlent de naissances anormales. Les plus anciens témoignages se trouvent dans les traités de la collection hippocratique. L'auteur des Epidémies mentionne ainsi la naissance d'un fœtus sans squelette $(2,19)$, et d'un enfant dont le bras droit était soudé au corps $(5,13)^{38}$. L'auteur du traité De la Génération (10) ne décrit pas de cas particulier, mais il tente d'expliquer l'origine des anomalies physiques chez le nouveau-né. Il attribue la présence de malformations à un problème survenu pendant la gestation. L'enfant peut avoir souffert d'un traumatisme subi par sa mère et naître estropié à l'endroit de la contusion. Le fœtus peut également avoir manqué d'espace dans la matrice et naître estropié à l'endroit qui a été comprimé: «C'est comme les arbres qui dans la terre n'ont pas d'espace, mais sont arrêtés par une pierre ou autre chose: en poussant, ils sont tortus, gros d'un côté, minces de l'autre» ${ }^{39}$.

Dans le livre 4 de la Génération des Animaux, Aristote pose les fondements de la tératologie moderne en passant systématiquement en revue les différentes formes de monstruosités animales et humaines et en tentant d'expliquer leur formation ${ }^{40}$. Il place dans la même catégorie les anomalies caractérisées par une déficience ou la présence de parties supplémentaires: «il naît parfois certains petits avec des doigts en surnombre, d'autres avec un seul, et

genetik 3, 1967, 244-263. Les auteurs relèvent que dans plusieurs cas un membre se développe normalement $\left(\mathrm{n}^{\text {os }} 4,15,27,36,45,57\right)$.

38 Epidémies, 2,19 = Littré 5, 93; 5,13 = Littré 5, 213.

39 Voir aussi De la Génération $9=$ Littré 7, 483.

40 P. Louis, «Monstres et monstruosités dans la biologie d'Aristote», in J. Bingen, G. Cambier, G. Nachtergael (eds.), Le monde grec. Hommages à Claire Préaux, Bruxelles, 1978, 277-284; H. Balss, «Die Zeugungslehre und Embryologie in der Antike. Eine Übersicht», Quellen und Studien zur Geschichte der Naturwissenschaften und der Medizin 5, 1936, 72-73; R. Garland, The eye of the beholder. Deformity and disability in the Graeco-Roman world, London, 1995, $152-156$. 
ainsi de suite pour les autres parties: certains ont des parties en trop, certains sont mutilés» $(4,4,770$ b $30-33)$. Ces anomalies peuvent toucher les organes internes: «certains font défaut ou sont tronqués ou sont en surnombre ou ne sont pas à leur place» (770 b 37-771 b 1-2). Aristote relève que les êtres atteints de telles malformations sont viables, sauf si «l'anomalie touche les parties vitales» $(4,4,771$ a 11-14). Comme Hippocrate, il fait remonter l'origine de telles malformations à l'époque de la gestation. Tandis que la production de parties surnuméraires est attribuée à la même cause que la formation de jumeaux, c'est-à-dire à un surplus de matière séminale $(4,4,772 \mathrm{~b}$ 13-773 a 13), l'absence d'une partie ou d'un membre entier est assimilée à un avortement, peut-être causé par une alimentation imparfaite de l'embryon ${ }^{41}$; l'embryon peut aussi être malformé ou mutilé à cause d'un défaut de température du résidu spermatique ${ }^{42}$. On notera que pour Aristote les naissances monstrueuses sont contraires à la nature ( $\pi \alpha \rho \dot{\alpha} \phi v ́ \sigma ı v)$, mais «à la nature considérée non pas dans sa constance absolue, mais dans son cours ordinaire: car du point de vue de la nature éternelle et soumise à la nécessité, rien ne se produit contre nature» $(4,4,770$ b 9-11). La tradition annalistique romaine mentionne plusieurs cas de naissances d'enfants malformés. TiteLive signale ainsi la naissance d'un enfant avec une seule main à Sinuessa en 174 av. J.-C. et à Arretium en 192 av. J.-C., tandis que Julius Obsequens rapporte celle d'une petite fille sans mains à Privernum en 163 av. J.-C. ${ }^{43}$ L'opinion populaire voyait peut-être dans ces naissances l'effet d'«impressions» maternelles, une croyance encore longtemps présente dans l'inconscient collectif. I. Geoffroy Saint-Hilaire raconte ainsi à propos d'un nouveauné hémimèle que «la mère attribuait la singulière conformation de son enfant à des statues mutilées, dont la vue l'avait frappée pendant sa grossesse» ${ }^{44}$.

La rareté des témoignages scientifiques et médicaux relatifs aux phénomènes tératologiques est probablement due au manque d'intérêt des médecins antiques pour les pathologies infantiles de manière générale. $\mathrm{Ce}$ désintérêt tient en partie au fait que les accouchements étaient généralement pratiqués par une sage-femme, ou une femme expérimentée, qui procédait à

$414,4,772$ b 35-38; 4,4,746a 8-11. La notion d'«avortement des membres» est reprise par Geoffroy Saint-Hilaire (n. 36 supra), qui invente le terme d'ectromélie en le formant avec $\dot{\varepsilon} \kappa \tau \rho \omega \omega$ j'avorte et $\mu \dot{\varepsilon} \lambda o \varsigma$, membre; voir p. ex. 200, 206-237.

42 2,743a 27-30. Cette théorie remonte peut-être à Empédocle; cf. H. Diels, Fragmente der Vorsokratiker ${ }^{5}$ Berlin, 1934, 31 A 81, 1. 12-14.

43 Tite-Live 35,21 et 41,21; Julius Obsequens, Livre des Prodiges, ch. 14. Voir aussi ibid. ch. 5: une mule avec 3 pattes (182 av.); ch. 20: un garçon avec 3 pieds et une main (147 av.).

44 Geoffroy Saint-Hilaire (n. 36 supra) 214-215. Cf. I. Opie, M. Tatem, A Dictionary of superstitions, Oxford, 1989, 317-318. Sur la théorie des impressions maternelles dans l'Antiquité, voir D. Gourevitch, «Se mettre à trois pour faire un bel enfant, ou l'imprégnation par le regard», L'évolution psychiatrique, 52, 1987, 559--563; Garland (n. 40 supra) 151. 
la sélection des enfants viables. Les nouveau-nés atteints d'une grave malformation disparaissaient rapidement, soit de manière naturelle (le taux de mortalité infantile était très élevé), soit volontairement, par infanticide, avant d'avoir pu être examinés par un médecin ${ }^{45}$.

Les sources iconographiques ont conservé la trace d'anomalies congénitales, mais les représentations d'infirmités physiques sont très rares dans l'art grec archaïque et classique.

Les représentations des Molionides (ou Actorides) constituent l'une des exceptions les plus remarquables. Selon la légende, les Molionides sont deux frères jumeaux, issus d'un père divin, Poséidon, et d'un père mortel, Actor. Leur singularité est d'être des jumeaux siamois; les textes les décrivent comme formant un être double, uni à la taille, avec deux têtes, deux torses, et quatre membres, ce qui en fait des guerriers redoutables, doués d'une force exceptionnelle ${ }^{46}$. Les artistes grecs les figurent généralement soudés au niveau du torse (thoracopages) ou des hanches (pygopages). Si leur identification peut prêter à discussion sur la peinture de vase ${ }^{47}$, leur pathologie est clairement indiquée sur les objets en bronze gravés. Sur une fibule en bronze conservée à Athènes, les Molionides combattent Héraclès; on voit nettement leurs deux têtes et leur tronc commun muni de quatre bras et quatre jambes $^{48}$. Les Molionides peuvent être également représentés unis au niveau du bassin, comme sur le socle d'un cheval en bronze conservé à Londres ${ }^{49}$. Ces images de jumeaux siamois n'apparaissent qu'à l'époque géométrique, de 750 av. J.-C. au début du VII ${ }^{\mathrm{e}}$ s. av. J.-C. Les Molionides sont ensuite complètement absents des arts figurés.

Quelques vases archaïques et classiques dépeignent des personnages légendaires avec des anomalies physiques, comme le dieu Hephaïstos, affligé d'un ou deux pieds bots, le vieux Géras au corps émacié et décrépi, des satyres obèses, bossus ou acromégaliques ${ }^{50}$. Quant aux monstres de la mythologie, ils sont généralement figurés comme des créatures hybrides, mêlant l'humain et le bestial, qui échappent à toute analyse médicale. Les seuls êtres malformés qui apparaissent dans des scènes de la vie quotidienne sont les nains, essen-

45 Grmek (n. 21 supra) 25-26; Garland (n. 40 supra) 141-142. Cf. infra n. 54.

46 Hesiod, Catalogue des femmes, fr. 18 (ed. R. Merkelbach, M. L. West, Fragmenta Hesiodea, Oxford, 1967); Apollod., Bibl. 2,7,2. Voir R. Hampe, s.v. Aktorione, LIMC I, 1981, 472.

47 Voir p. ex. Hampe,ibid., 476; J. Boardman, «Symbol and story in geometric art», in W.G. Moon (ed.), Ancient Greek art and iconography, Madison and London, 1983,25-26.

48 Cf. les photographies de jumeaux siamois in M. Monestier, Les monstres, Paris, 1981,282-304.

49 Cheval en bronze de Phigalia; Londres, British Museum (vers 750 av.); Hampe (n. 46 supra) $\mathrm{n}^{\circ} 1$ (fig.).

50 Hephaïstos: F. Brommer, Hephaistos. Der Schmiedegott in der antiken Kunst, Mainz am Rhein, 1978. Géras: H. A. Shapiro, s.v. Geras, LIMC, IV, 1988, 180-182. Satyres: voir p. ex. J. Boardman, Athenian red figure vases. The archaic period, London, 1975, figs 83 et 116. 
tiellement des achondroplastes. On notera que l'étrangeté du nain n'est pas figurée comme une monstruosité: les nains sont assimilés aux satyres, ce qui rend leur anomalie tolérable ${ }^{51}$. La parenté de l'estropié de Genève avec les comastes accroupis, souvent dotés d'attributs dionysiaques, n'est peut-être pas due au hasard; elle pourrait indiquer que son anomalie, comme celle des nains, était associée au monde hors norme de Dionysos ${ }^{52}$.

La paléopathologie offre quelques cas comparables, en particulier celui d'un squelette découvert en Grande-Bretagne dans un cimetière datant de la fin du VI ${ }^{\mathrm{e}}$-début du VII ${ }^{\mathrm{e}} \mathrm{s}$. apr. J.-C. Ce squelette appartenait à un homme âgé d'environ 28 à 30 ans; il était dépourvu des os du membre supérieur gauche, ainsi que des os de la ceinture scapulaire (la clavicule et l'omoplate). La tombe n'avait pas été profanée, le squelette était parfaitement conservé, et $\mathrm{S}$. Chadwick Hawkes et $\mathrm{C}$. Wells ont pu démontrer que l'homme était atteint d'une malformation congénitale. Le développement compensatoire d'autres os, en particulier de la colonne vertébrale (scoliose), et la présence d'une exostose déformant la cheville droite, confirment que l'anormalité était ancienne $\mathrm{e}^{53}$.

\section{Aspects socio-religieux}

L'estropié du Musée d'art et d'histoire de Genève soulève d'une part la question des conditions de survie des enfants malformés en Grèce antique (a), d'autre part celle de la fonction et de la signification de cet objet dans un contexte funéraire (b).

\section{a) L'élimination des enfants malformés}

Diverses sources attestent qu'en Grèce l'exposition d'un nouveau-né physiquement anormal était légalement admise, mais avec des variantes importantes selon les Etats ${ }^{54}$. A Athènes, par exemple, une telle décision relevait de la sphère privée. Elle devait être prise rapidement, dans la semaine suivant l'accouchement, avant la célébration de la fête des Amphidromies

51 Voir V.Dasen, Dwarfs in ancient Egypt and Greece, Oxford, 1993, en particulier 173,236-241.

52 La mise en évidence du sexe de l'estropié est un indice qui va dans le même sens; cf. supra n. 13.

53 S. Chadwick Hawkes, C. Wells, «Absence of the left upper limb and pectoral girdle in a unique Anglo-Saxon burial», Bull. N.Y. Acad. Med. 52/10, 1976, 1229-1235. Voir aussi Ortner/ Putschar (supra n. 30) 362-364 (malformations des extrémités).

54 E. Eyben, «Family planning in Graeco-Roman antiquity», Ancient Society 11/12, 1980/1981, 5-82; M. Schmidt, «Hephaistos lebt - Untersuchungen zur Frage der Behandlung behinderter Kinder in der Antike», Hephaistos 5/6, 1983/1984, 133-161; Garland (n. 40 supra), 13-18, $65-72$. 
qui marquait l'entrée officielle de l'enfant dans la communautés5. A Sparte, par contre, une loi attribuée à Lycurgue ordonnait que le nouveau-né soit examiné par le conseil des Anciens. S'il était faible ou malformé, il devait être déposé aux abords d'un précipice sur le Mont Taygète, où il n'avait aucune chance de survivre ${ }^{56}$. Le but de cette législation était eugénique: dans cet état soumis à un idéal militaire, il fallait préserver la supériorité de l'élite dirigeante qui ne devait compter que des membres physiquement parfaits. Mais de telles mesures n'ont pas d'égal ailleurs en Grèce ${ }^{57}$.

Quels étaient les critères d'élimination du nouveau-né? Un examen du vocabulaire ne nous donne pas de réponse précise. Les termes utilisés sont vagues: chez Platon, les enfants indésirables sont désignés par le terme

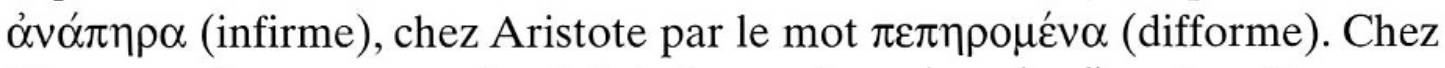

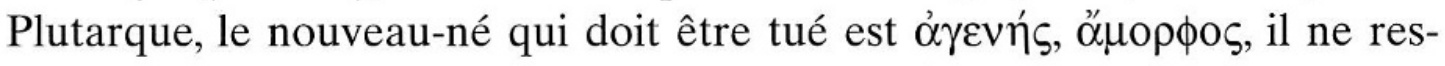
semble pas à ses parents, il est donc monstrueux ${ }^{58}$.

Divers documents montrent qu'en pratique les parents n'éliminaient pas systématiquement les nouveau-nés dont l'apparence physique était anormale. Des anomalies légères étaient tolérées ${ }^{59}$. Les enfants atteints de graves malformations, privés de membres ou avec des membres surnuméraires, sont généralement mort-nés ou ne vivent que quelques heures; mais les plus robustes peuvent échapper à la mort ${ }^{60}$. Il est donc concevable que l'estropié de Genève ait été soit élevé par ses parents, soit exposé puis recueilli par une personne mue par la pitié ou la curiosité. Comme l'hémimélie n'est pas associée à une malformation des organes internes, l'enfant a pu survivre et atteindre l'âge adulte sans nécessiter de soins médicaux particuliers.

\section{b) Fonction et signification de l'objet}

Le contexte précis de la trouvaille n'est pas connu et de nombreuses questions restent sans réponse. A qui appartenait la tombe qui contenait la figurine? A l'estropié lui-même? Ou à un membre de sa famille, à son protecteur ou à son maître?

Aucun indice ne permet de deviner le statut social de notre personnage. G. Python suggère que des pouvoirs surnaturels lui furent peut-être conférés

55 La cérémonie avait lieu 5,7 ou 10 jours après la naissance. Voir M. Golden, Children and childhood in classical Athens, Baltimore and London, 1990, 23-24.

56 Plutarque, Lycurgue 16,1-2.

57 On retrouve l'esprit eugénique de la législation laconienne dans les doctrines de Platon et d'Aristote: Plat. Rep. 460; Arist. Pol. 7,14,1335b.

58 Ces termes apparaissent également dans les serments. Voir p. ex. Eschine 3,111.

59 Cf. p. ex. les représentations de nains dans l'iconographie archaïque et classique; Dasen (n. 51 supra). Ou la forme inhabituelle du crâne de Périclès; Plutarque, Périclès 3,3-4.

60 Pour les jumeaux siamois, cf. la figure légendaire des Molionides (n. 46-49 supra). 
à cause de son handicap, et qu'il s'agissait d'un devin ${ }^{61}$. Les sources littéraires mentionnent en effet quelques devins atteints d'infirmité, en particulier de cécité, comme Tirésias ou le Thrace Phinée. Mais la cécité est un handicap d'une nature particulière qui permet à celui qu'elle frappe d'établir un rapport privilégié avec le monde divin ${ }^{62}$. Aucun devin ne semble avoir eu une autre anomalie physique importante. Au contraire, une infirmité physique semble avoir été incompatible avec l'exercice d'une fonction religieuse. A Athènes, par exemple, un invalide ( $\alpha \dot{\delta} v \dot{v} \alpha \tau \sigma \varsigma$ ) ne pouvait pas prendre part au tirage au sort des archontes. L'Etymologicum Magnum précise que celui qui voulait exercer la fonction de prêtre ou de $\beta \alpha \sigma i \lambda \varepsilon v ́ \varsigma$ devait être

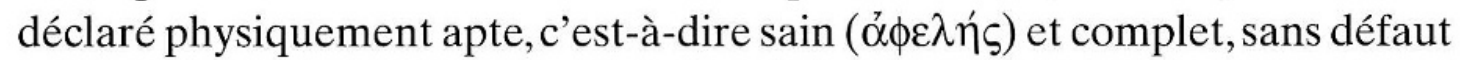

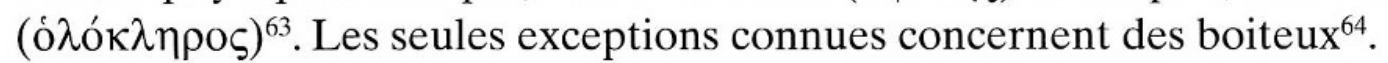

Un élément de réponse réside peut-être dans le réalisme de la figurine. Il est tentant de mettre en rapport la précision du coroplaste avec l'intérêt pour la physiologie des philosophes de la nature présocratiques, et en particulier des penseurs de Grande Grèce ${ }^{65}$. Ce n'est peut-être pas un hasard si le vase plastique a été découvert en Sicile où existait une influente école médicale à l'époque archaïque. Le plus ancien témoignage archéologique grec relatif à un médecin provient de Mégara Hyblaea:il s'agit d'un couros dédié au médecin Sombrotidas en 580-570 av. J.-C. ${ }^{66}$ La Sicile a compté plusieurs médecins célèbres, comme Démocédès de Crotone (vers 500 av. J.-C.), médecin attitré de Polycrate de Samos, puis de Darius (Hérodote 3,129-136), souvent aussi philosophes, comme Alcméon de Crotone, élève de Pythagore (vers 500 av. J.-C. $)^{67}$. La terre cuite de Genève a peut-être été commanditée par un de ces hommes, penseur ou médecin, désireux de conserver un témoignage de ce phénomène avec le plus d'exactitude possible; la figurine était peutêtre placée dans sa tombe, à la façon des Egyptiens qui aimaient être enterrés avec l'effigie de leur nain favori.

Il est intéressant de noter que cet objet si exceptionnel a été obtenu au moule et qu'il a été reproduit. Le lieu de trouvaille du second exemplaire est

61 Python (n. 1 supra).

62 A. Esser, Das Antlitz der Blindheit in der Antike, Leiden, 1961, 99-104; R. G. A. Buxton, «Blindness and limits: Sophokles and the logic of myth»,Journal of Hellenic Studies 100,1981, 22-37.

63 Lysias 24, 13; Etym. m. s.v.'Aфع $\lambda$ ń. Cf. Dasen (n. 51 supra) 211-212.

64 Comme p. ex. le roi Medon, le premier archonte d'Athènes (Paus. 7,2,1) ou le devin Hégésistrate (Hdt. 9,37).

65 H. E. Sigerist, $A$ History of medicine, II, New York, 1961; F. Kudlien, Der Beginn des medizinischen Denkens bei den Griechen, Zürich und Stuttgart, 1967. Cf. Ph. Mudry, La préface du De Medicina de Celse, Lausanne, 1982, 60-62.

66 E. Berger, Das Basler Arztrelief, Basel, 1970,155, figs 162-163.

67 M. Michler, «Das Problem der westgriechischen Heilkunde», Sudhoffs Arch. 46, 1962, 137-152; Berger (n. 66 supra) 152-153. 
malheureusement inconnu: provient-il également de la nécropole, ou du site de la ville antique? Etait-il destiné à l'estropié lui-même?

Pour conclure, signalons qu'un document de 480 av. J.-C. associe également un médecin à un personnage atteint d'une anomalie congénitale. L'aryballe Peytel, conservé au Musée du Louvre, figure en effet l'officine d'un jeune médecin. Sur l'une des faces du vase, le médecin est assis en train de pratiquer une saignée; sur l'autre face, un nain, peut-être son serviteur, s'entretient avec un patient en portant un lièvre. Or les aryballes sont des pots à onguents, comme les vases plastiques du type des comastes ${ }^{68}$.

\section{Remerciements}

Je tiens à exprimer toute ma gratitude à M. Jacques Chamay du Musée d'art et d'histoire de Genève qui m'a permis de publier cet objet, ainsi qu'à l'Association des Amis de l'Université de Fribourg qui a soutenu financièrement cette recherche.

Mes remerciements vont également aux Professeurs C. A. Baud et R. Lagier à Genève (CMU Département de pathologie), T. Pexieder à Lausanne (Institut d'histologie et d'embryologie) et P. Sprumont à Fribourg (Institut d'anatomie) pour leur aimable collaboration.

68 Dasen (n. 51 supra) 229-230,233-234, pl. 44. Mais la terre cuite de Genève n'a pas de trous de suspension, contrairement à la plupart des vases de comastes. 